\title{
Wavelet-threshold based Bit Intensity Measurement: On Facial Expression Recognition
}

\author{
Tanjea Ane \\ Lecturer, dept. of ETE, The People's University of Bangladesh \\ Dhaka: 1207, Bangladesh
}

\begin{abstract}
The objective of this paper is to examine the threshold concept for facial expression recognition, in facial image which is mostly preferred to represent facial expressions in computer vision applications, including face recognition and expression analysis. The goal of this study, bit intensity measurement with thresholding method is applied on facial image for expression recognition. Each image partitioned into equal regions from which the feature binary attributes are extracted and integrated into a feature vector to be used as a face descriptor. Binary measurement has been done matching with reference and neighbouring pixels. Extracted local binary features are integrated for sorting into thresholding based similarity index according to their wave-threshold coefficient values. For evaluation the proposed descriptors JAFFE dataset, Support Vector Machine, Cross validation, fdlibmex were applied.
\end{abstract}

\section{Keywords}

Feature descriptor, soft wavelet threshold, similarity index, JAFFE.

\section{INTRODUCTION}

In this section a brief review of previous approached are described. Facial actions are most commonly used in human behavior analysis. Facial expression is a key integral section of human to human contact. There are few lists of principal facial emotions so far surprise, anger, disgust, contempt, fear, sadness, happiness. Automated perception of facial expression is implemented for intercommunication in various sites [1], [2]. Natural system composes vital seven candidate feelings [3].In [4], facial expression recognition (FER) represents genetic programming based method to search the better classification percentage and fitness power with composite operator which makes it complex.

In human communication, only $7 \%$ is assigned by the verbal part, $38 \%$ by facial movement but $55 \%$ by facial expression [5]. Capturing facial points from both forward and sideward view points are mentioned as crucial for facial features (e.g., face, lip, view, nose) [6]. Most of researches have been done on dynamic textures [7, 8, 9, 10, 11, 12, 13]. [14] Study showed to accept fine grained diversity in facial expression on the basis of Facial Action Coding System (FACS) and action units (AUs).

In [15], for building a model of human facial expressions from moving segment as input image Hidden Markov Models (HMMs) was proposed. Binary local features were deal with primary image features captured around the sides of eyes, nose and mouth [16], [17]. Rule based reasoning and feature extraction were applied to seize AUs [18]. Space and time were regarded with facial expression recognition in paper [19]. For each pixel the descriptor selects a label to every pixel of an image by threshold neighbourhood of each pixel with the centre pixel value and considering the outcome as a binary number. Then the histogram of the different layers can be considered as a feature vector. Multiresolution approach and rotation invariant texture analysis showed in ref. paper [20].

Facial representation method based on Local Binary Pattern (LBP) was proposed [21]. [22] Paper proposed for facial mood recognition, candied grid nodes to make facial wire frame model. These papers experimented on geometrybased extraction process. It was rotated-invariant system and here feature vector length was not lengthy. LBP is justified to be an impressive texture descriptor and practiced for pattern recognition research work e.g., facial expression recognition [23],[24]. Both local binary pattern and local phase quantization method were applied but system is time consuming for a facial expression recognition system in [25]. In [26], examined a texture descriptor LPQ (Local Phase Quantization). Image recognition method studied invariant with shifting and altering described in [27].

A study paper for feature representation method can hold data from local pixels segment. It examines a pixel relations of the referenced pixel ('c') with four pixels at level one ('a1','a2','a3'and'a4') and different four pixels at level two ('b1','b2','b3','b4') using algorithm of LDGP (Local Distinctive Gradient Pattern) [28] for boosting the facial expression recognition model. In this paper proposed a new wave coefficient system using an alternative feature extraction method while feature vector is designed with soft waveletthreshold measurement.

\section{SYSTEM IMPLEMENTATION}

The most significant segment of selection the feature vector is a pattern classification. Better accuracy in classification depends on feature extraction process. Process includes neighbourhood values are thresholded compared with the center(reference) value and the outcome is binary number. In this way, skillful encoding applied to read the neighbourhood information. The values in $5 \times 3$ neighborhoods are thresholded and their binary attributes intensity give the feature value at local pixel blocks. The feature data is owned by partitioning the facial image into several small blocks, then the attributes of each small region is found, and finally all these features are concatenated to give a global descriptor. The whole image results in three levels global features, region features and pixel level features so that reduces the loss of spatial information. Table 1, has shown detail demo for achieving the feature pattern while the reference pixel is larger than neighbourhood pixel, the binary value sets zero otherwise one for each pixel relation. 
Table 1: Binary pattern extraction with example for two levels(Pattern 1, Pattern 2)
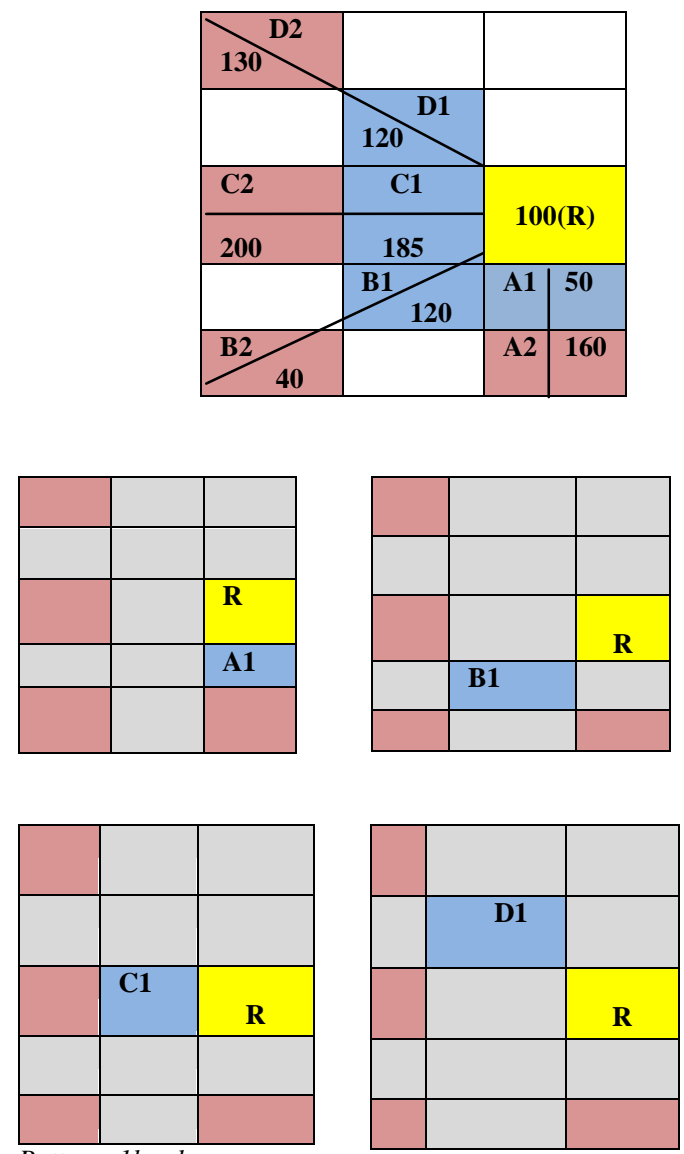

Pattern-1level

$1^{\text {st }}$ pattern $=0111$
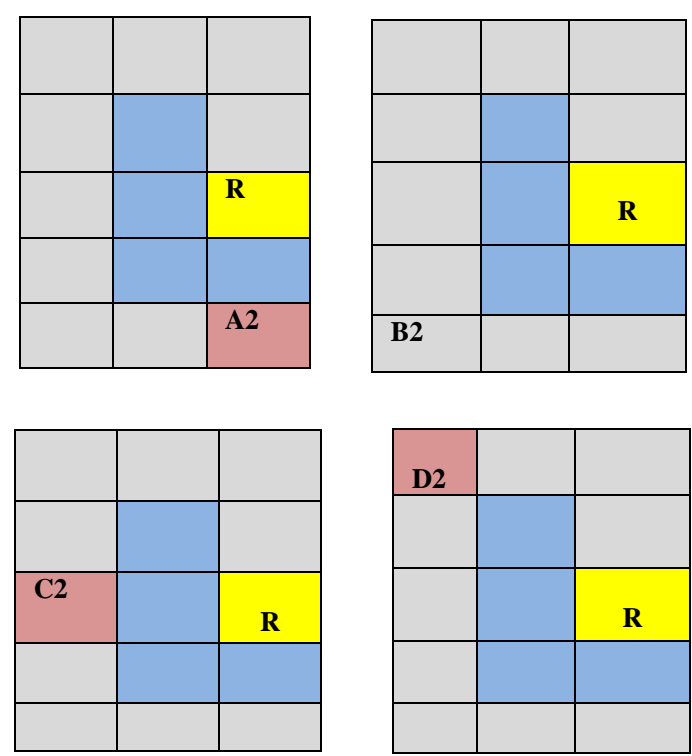

\subsection{Wavelet Coefficient Based Feature}

Facial image expression to be retrieved while sending the facial signal $f$ with zero mean, standard deviation $\boldsymbol{n}_{i j}$ the noisy measurement $g_{i j}=f_{i j}+\sigma n_{i j}$ is attained from the initial face of $\mathrm{M} \times \mathrm{M}$ matrix considers $\boldsymbol{f}=\left\{f_{i j}, \mathrm{i}, \mathrm{j}=1,2 \ldots \mathrm{M}\right\}$. From [31] paper refers,

$T_{s}=\left\{\begin{array}{lrr}\frac{\sigma_{y}^{2}}{\sigma_{x}^{2}} \quad \text { if } & \sigma_{v}{ }^{2}<\sigma_{y}{ }^{2} \\ \max \left\{\left[A_{m}\right]\right\} & \text { otherwise }\end{array}\right.$

Thresholding is very efficient method which achieves on wavelet coefficient by comparing with threshold which is computed using shrinkage procedures. If the coefficient is lower than the threshold it is set to zero else it is adjusted. Altering the low noisy-coefficients by zero and the result provides reconstruction attributes without noise with mean squared error is reduced. The wavelet soft thresholding technique has been applied over hard thresholding, because it outcomes nice images as compared to hard thresholding. Bayes shrink is calculated [31, 32] as follows: where $\mathrm{X}$ and $\mathrm{V}$ are mutually independent, the variances $\sigma_{y}^{2}$ for noisy image, $\sigma_{x}^{2}$ for original image and $\sigma_{v}^{2}$ for noise,

$$
\sigma_{y}^{2}=\sigma_{x}^{2}+\sigma_{v}^{2}
$$

$Y=X+V$

$Y$ is the wavelet transforms of the degraded image, $X$ is the wavelet transform of the original image, and $V$ is the wavelet transform of the noise components.

\subsection{Binary Classification}

Support Vector Machine (SVM) algorithm constructs a margin that separates different class labels so that the space among different classes is as massive as possible. In linear SVM given a training dataset of points of the pattern $\left(\mathrm{x}_{1}, \mathrm{y}_{1}\right), \ldots . .\left(\mathrm{x}_{\mathrm{n}}, \mathrm{y}_{\mathrm{n}}\right)$ where the $\mathrm{y}_{\mathrm{i}}$ are either 1 or -1 , each illustrating the class to which the point $x_{i}$ exists. SVM intersects the group of points $x_{i}$ for which $y_{i}=1$ from the group of points for which $y_{i}=-1$, which is defined so that the space between the margin can be written as the set of points modifying data points from falling into the margin, the marginal function follows the $(4,5,6)$ equations.

$w \cdot x+b \geq 1$, if $y_{i=1}$

or

$w \cdot x+b \leq=-1$, if $y_{i}=-1$

Multiclass SVM separate the training set with maximal margin. If $s_{i}$ the support vectors, $\alpha_{i}$ are the weights, $b$ is the bias, and $k_{\mathrm{e}}$ denotes linear kernel function, the svm results svmtrain to separate the vectors $x$ with following equation:

$c=\sum_{i} \alpha_{i} k_{e}\left(s_{i}, x\right)+b$

(6)

\section{EXPERIMENT AND RESULT ANALYSIS}

Experiments are applied on JAFFE dataset; with 213 images while seven expression (Happy, sad, fear, disgust, anger, contempt, surprise) classes. Here used 'fdlibmex' matlab library $[29,30]$ to detect frontal face that made a dimension of 
face in 99x99 pixels. Each local block has a dimension of $11 \times 11$ pixels. For facial identification "fdlibmex " matlab library is applied and no other simulation are needed, so its usage is very efficient. The mex file that outcomes the frontal face from input image.

Table 2: Confusion matrix for expression classes

\begin{tabular}{|l|l|l|l|l|l|l|l|}
\hline $\begin{array}{l}\text { Predict- } \\
\text { ion }\end{array}$ & $\begin{array}{l}\text { Angr } \\
\text { y }\end{array}$ & Disgust & Fear & $\begin{array}{l}\text { Hap } \\
\text { py }\end{array}$ & $\begin{array}{l}\text { Neutr } \\
\text { al }\end{array}$ & Sad & $\begin{array}{l}\text { Surp } \\
\text { rise }\end{array}$ \\
\hline Angry & $\mathbf{1 0 0}$ & $\mathbf{0 . 0}$ & $\mathbf{0 . 0}$ & $\mathbf{0 . 0}$ & $\mathbf{0 . 0}$ & $\mathbf{0 . 0}$ & $\mathbf{0 . 0}$ \\
\hline Disgust & $\mathbf{4 . 9}$ & $\mathbf{9 4 . 1}$ & $\mathbf{1 . 0}$ & $\mathbf{0 . 0}$ & $\mathbf{0 . 0}$ & $\mathbf{0 . 0}$ & $\mathbf{0 . 0}$ \\
\hline Fear & $\mathbf{0 . 0}$ & $\mathbf{0 . 0}$ & $\mathbf{9 0 . 4}$ & $\mathbf{9 . 6}$ & $\mathbf{0 . 0}$ & $\mathbf{0 . 0}$ & $\mathbf{0 . 0}$ \\
\hline Happy & $\mathbf{0 . 0}$ & $\mathbf{0 . 0}$ & $\mathbf{0 . 0}$ & $\mathbf{9 9 . 0}$ & $\mathbf{1 . 0}$ & $\mathbf{0 . 0}$ & $\mathbf{0 . 0}$ \\
\hline Neutral & $\mathbf{0 . 0}$ & $\mathbf{0 . 0}$ & $\mathbf{0 . 0}$ & $\mathbf{0 . 0}$ & $\mathbf{1 0 0}$ & $\mathbf{0 . 0}$ & $\mathbf{0 . 0}$ \\
\hline Sad & $\mathbf{0 . 0}$ & $\mathbf{0 . 0}$ & $\mathbf{0 . 0}$ & $\mathbf{0 . 0}$ & $\mathbf{0 . 0}$ & $\mathbf{9 8 . 7}$ & $\mathbf{1 . 3}$ \\
\hline Surprise & $\mathbf{0 . 0}$ & $\mathbf{0 . 0}$ & $\mathbf{0 . 0}$ & $\mathbf{0 . 0}$ & $\mathbf{0 . 0}$ & $\mathbf{2 . 4}$ & $\mathbf{9 7 . 6}$ \\
& & & & & & & \\
\hline
\end{tabular}

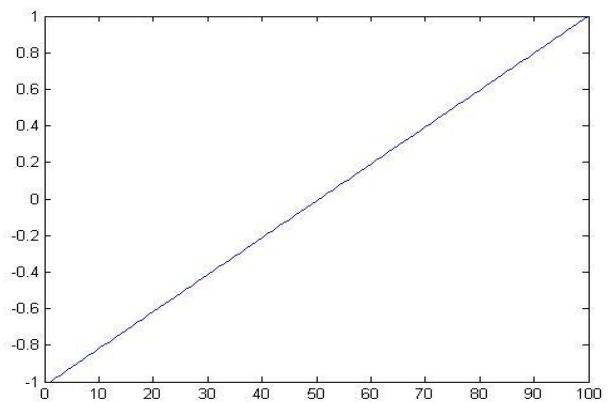

Figure 1: Original pattern extraction

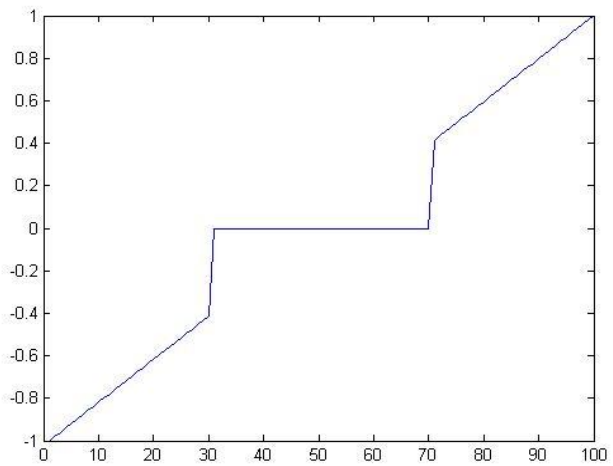

Figure 2: Hard thresholding feature extraction

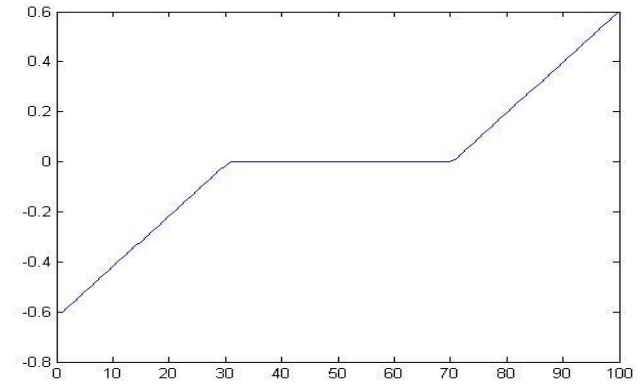

Figure 3: Proposed Soft thresholding feature extraction

Table 3: Seven expression classes

\begin{tabular}{c|c|}
\cline { 2 - 2 } Expression class & JAFFE \\
\hline Anger & 30 \\
\hline Disgust & 29 \\
\hline Fear & 32 \\
\hline Happy & 31 \\
\hline Sadness & 31 \\
\hline Surprise & 30 \\
\hline Neutral & 30
\end{tabular}

Table 4: Comparison view of facial expression recognition

\begin{tabular}{|c|c|c|}
\hline Method & Classifier & $\begin{array}{c}\text { Accuracy } \\
(\boldsymbol{\%})\end{array}$ \\
\hline $\begin{array}{c}\text { Soft Wavelet- } \\
\text { thresholding } \\
\text { method }\end{array}$ & $\begin{array}{c}\text { Multiclass } \\
\text { SVM(Poly) }\end{array}$ & 93.50 \\
\hline $\begin{array}{c}\text { Local binary } \\
\text { pattern }\end{array}$ & SVM & 90.42 \\
\hline $\begin{array}{c}\text { Gradient Direction } \\
\text { Pattern }\end{array}$ & $\begin{array}{c}\text { Multiclass } \\
\text { SVM(Poly) }\end{array}$ & 92.11 \\
\hline Gabor filter & $\mathrm{NN}$ & 90.10 \\
\hline
\end{tabular}


Table 5: Classification accuracy and processing time comparison

\begin{tabular}{|c|c|c|c|}
\hline Method & Classification & $\begin{array}{c}\text { Feature } \\
\text { extraction } \\
\text { time(sec) }\end{array}$ & $\begin{array}{c}\text { Classification } \\
\text { time(sec) }\end{array}$ \\
\hline $\begin{array}{c}\text { Proposed } \\
\text { soft wavelet } \\
\text {-coefficient } \\
\text { method }\end{array}$ & $93.50 \%$ & 0.001 & 0.003 \\
\hline LPQ & $79.11 \%$ & 0.095 & 0.070 \\
\hline LBP & $90.42 \%$ & 0.026 & 0.070 \\
\hline $\mathbf{L B P}_{\mathbf{U} 2}$ & $84.43 \%$ & 0.039 & 0.025 \\
\hline $\mathbf{L B P}_{\mathbf{u} 2}+\mathbf{L P Q}$ & $83.82 \%$ & 0.137 & 1.00 \\
\hline
\end{tabular}

\section{CONCLUSION}

Paper surveying, proposed system shows a significant improvement for achieving facial features. A smooth and competent facial expression illustration and classification approach is analyzed here. Facial expression representations are based on LBP, SLBM and MBWM features; features are analyzed using the multiclass support vector machine, which are complicated and rich. Newly proposed system is experimented with seven universal expressions i.e. anger, disgust, fear, happy, sad, neutral, fear. In this work image thresholding scheme based on wavelet threshold was implemented using MATLAB platform. Bayes shrink rule combined with soft thresholding function was applied to the test image with gaussian noise. It clearly identified the facial expression without any confusion among the expression classes, improves misclassification results among the expression classes and achieved accuracy $\sim 94 \%$. From above discussion, new thresholding bit intensity based technique executes with lower processing time, higher performance and accuracy skill.

\section{REFERENCES}

[1] I. S. Pandzic and R. Forchheimer, Eds., MPEG-4 Facial Animation. New York: Wiley, 2002.

[2] P.Ekman and W.Friesen,Facial Action Coding System,Palo Alto,CA Consulting Psychologists Press,1978.Masana, R., Daqaq, M.F.: Electromechanical modeling and nonlinear analysis of axially loaded energy harvesters. Journal of Vibration and Acoustics. 133, 011007-1 (2011)

[3] J. A. Russell and J. M. Fernandez-Dols, The Psychology of Facial .Expression. Cambridge,U.K.: Cambridge Univ. Press, 1997.

[4] Guodong Guo, Stan Z. Li, and Kapluk Chan," Face Recognition by Support Vector Machines", School of Electrical and Electronic Engineering Nanyang Technological University, Singapore 639798

[5] A. Mehrabian, Communication without words, psychology Today. 2(9) pp. 53-56, 1968.
[6] M. Pantic and L. Rothkrantz, "Automatic analysis of facial expressions: The state of the art," IEEE Trans. Pattern Anal. Mach. Intell., vol. 22, no. 12, pp. 1424 1445, Dec. 2000.

[7] J. Yu and B. Bhanu, "Evolutionary feature synthesis for facial expression recognition," Pattern Recog. Lett., vol. 27, no. 11, pp. 1289-1298,Aug. 2006.

[8] G. Zhao and M. Pietikäinen, "Dynamic texture recognition using local binary patterns with an application to facial expressions," IEEE Trans.Pattern Anal. Mach. Intell., vol. 29, no. 6, pp. 915-928, Jun. 2007.

[9] Y. Tong, J. Chen, and Q. Ji, "A unified probabilistic framework for spon-taneous facial action modeling and understanding," IEEE Trans.Pattern Anal. Mach. Intell., vol. 32, no. 2, pp. 258-273, Feb. 2010.

[10] T. Wu, M. Bartlett, and J. Movellan, "Facial expression recognition using Gabor motion energy filters," in Proc. IEEE Int. Conf. Comput. Vis. Pattern Recog. Workshop Human Commun. Behav. Anal., Jun. 2010, pp. 42-47.

[11] J. N. Bassili, "Emotion recognition: The role of facial movement and the relative importance of upper and lower areas of the face," Pers. SocialPsychol., vol. 37, no. 11, pp. 2049-2058, Nov. 1979.

[12] P. Yang, Q. Liu, and D. Metaxas, "Boosting coded dynamic features for facial action units and facial expression recognition," in Proc. IEEE Conf. Comput. Vis. Pattern Recog., Jun. 2007, pp. 1-6.

[13] Z. Ambadar, J. Schooler, and J. Cohn, "Deciphering the enigmatic face: The importance of facial dynamics to interpreting subtle facial expressions,"Psychol. Sci., vol. 16, no. 5, pp. 403-410, May 2005.

[14] I. Cohen, N. Sebe, A. Garg, L.S. Chen and T.S. Huang, "Facial expression recognition from video sequences: Temporal and static modeling," Comput. Vision Image Understanding, Vol. 91, pp. 160-187, August 2003.

[15] Y.L. Tian, T. Kanade and J.F. Cohn, "Recognizing action units for facial expressions analysis," IEEE Trans. Pattern Analysis Machine Intell.,Vol. 23, No. 2, pp. 97 115, March 2001.

[16] Y. Zhang and Q. Ji, "Active and dynamic information fusion for facial expression understanding from image sequences," IEEE Trans. Pattern Anal. Machine Intelli., vol. 27, pp. 699-714, May 2005.

[17] T. Anderson, A. Handid and M. Pietikainen, "Face description with local binary patterns: Application to face recognition," IEEE Trans. Pattern Anal. Mach. Intel., Vol. 28, pp. 2037-2041, Dec. 2006.

[18] M. Pantic, M. I. Patras, "Dynamics of facial expression: Recognition of facial actions and their temporal segments from face profile image sequences," IEEE Trans. Syst. Man Cybernet. Part B: Cybernet., Vol. 36: pp. 433-449, April 2006.

[19] Z. Yeasin, M. Pantic, G.I. Roisman and T.S. Huang, “A survey of affect recognition methods: Audio, visual and spontaneous expressions," IEEE Trans. Pattern Anal. Machine Intelli., Vol. 31, pp. 39-58, Jan. 2009.

[20] IEEE Trans. Pattern Anal. Mach. Intel., Vol. 28, pp. 2037-2041, Dec. 2006. 
[21] T. Ahonen, A. Hadid and M. Pietikainen, "Face description with local binary patterns: Application to face recognition".

[22] I. Kotsia and I. Pitas, "Facial expression recognition in image sequences using geometric deformation features and support vector machines,"IEEE Trans. Image Process., Vol. 16, pp. 172-187, Jan. 2007.

[23] T. Ojala, M. Pietikainen and T. Maenpaa, "Multiresolution Gray-scale and rotation invariant texture classification with local binary patterns," IEEE Trans. Pattern Analysis Machine Intellig., Vol. 24, pp. 971-987, July 2002.

[24] G. Zhao and M. Pietikainen, "Dynamic texture recognition using local binary patterns with an application to facial expressions," IEEE Trans.Pattern Anal. Mach. Intell., Vol. 29: pp. 915-928, June 2007.

[25] V. Ojansivu and J. Heikkila, "Blur insensitive texture classification using local phase quantization," Proceedings of the 3rd International Conference on Image and Signal Processing, July 1-3, 2008, CherbourgOcteville, France, pp. 236-243.

[26] L. Ma and K. Khorasani, "Facial expression recognition using constructive feed forward neural networks," IEEE Trans. Syst. Man Cybernet. Part B: Cybernet., Vol. 34, pp. 1588-1595, June 2004.
[27] S. Yang and B. Bhanu, "Understanding discrete facial expressions in video using an emotion avatar image," IEEE .

[28] MohammadShahidul Islam, Surapong Auwatanamongkol Md. Zahid Hasan," Boosting Facial Expression Recognition Using LDGP- Local Distinctive Gradient Pattern”, ICEEICT Conference Paper 2014.

[29] M.J.Lyons, M.Kamachi and J.gyoba,The Japanese female facial expression (JAFFE) dataset Available: www.kasrl.org/jaffe_download.html.

[30] LIBSVM -- A Library for Support Vector Machines, multi- class classification Online Available: http://www.csie.ntu.edu.tw/ cjlin/libsvm.

[31] Namrata Dewangan, Agam Das Goswam," Image Denoising Using Wavelet Thresholding Methods", International Journal of Engineering Sciences \& Management, ISSN: 2277-5528, April-June, 2012.

[32] S.Sulochana, R.Vidhya" Image Denoising using Adaptive Thresholding in Framelet Transform Domain", (IJACSA) International Journal of Advanced Computer Science and Applications, Vol. 3, No. 9, 2012. 